Article

\title{
On the Momentum Transported by the Radiation Field of a Long Transient Dipole and Time Energy Uncertainty Principle
}

\author{
Vernon Cooray ${ }^{1, *}$ and Gerald Cooray ${ }^{2}$ \\ 1 Department of Engineering Sciences, Uppsala University, Uppsala 751 21, Sweden \\ 2 Department of Clinical Neuroscience, Karolinska University Hospital, Stockholm 171 76, Sweden; \\ Gerald.Cooray@ki.se \\ * Correspondence: vernon.cooray@angstrom.uu.se; Tel.: +46-18-471-5809 \\ Academic Editor: Robert W. Talbot \\ Received: 26 October 2016; Accepted: 17 November 2016; Published: 23 November 2016
}

\begin{abstract}
The paper describes the net momentum transported by the transient electromagnetic radiation field of a long transient dipole in free space. In the dipole a current is initiated at one end and propagates towards the other end where it is absorbed. The results show that the net momentum transported by the radiation is directed along the axis of the dipole where the currents are propagating. In general, the net momentum $P$ transported by the electromagnetic radiation of the dipole is less than the quantity $U / c$, where $U$ is the total energy radiated by the dipole and $c$ is the speed of light in free space. In the case of a Hertzian dipole, the net momentum transported by the radiation field is zero because of the spatial symmetry of the radiation field. As the effective wavelength of the current decreases with respect to the length of the dipole (or the duration of the current decreases with respect to the travel time of the current along the dipole), the net momentum transported by the radiation field becomes closer and closer to $U / c$, and for effective wavelengths which are much shorter than the length of the dipole, $P \approx U / c$. The results show that when the condition $P \approx U / c$ is satisfied, the radiated fields satisfy the condition $\Delta t \Delta U \geq h / 4 \pi$ where $\Delta t$ is the duration of the radiation, $\Delta U$ is the uncertainty in the dissipated energy and $h$ is the Plank constant.
\end{abstract}

Keywords: dipole radiation; electromagnetic fields; classical electrodynamics; position momentum uncertainty principle; time energy uncertainty principle

\section{Introduction}

Electromagnetic radiation fields are associated not only with energy but also with a momentum [1,2]. Thus, any object that emits electromagnetic radiation will experience a momentum equal and opposite to the momentum transported by the electromagnetic radiation. The net momentum transported by the radiation depends on its directive properties. If the electromagnetic radiation is directed in one particular direction, then the electromagnetic radiation will transport a net momentum in that direction. If the spatial distribution of the emitted electromagnetic field has mirror symmetry with respect to the $x-y, x-z$, and $y-z$ planes, the net momentum transported by the radiation is zero. Here we consider a transient dipole where a current is initiated at one end and propagates towards the other end where it is absorbed. The electromagnetic fields radiated by such transient dipoles are neither directed in one particular direction nor completely symmetric with respect to the three-spatial axis. In the case of dipoles, the net momentum transported by the radiation depends on the directivity of the radiation. The directivity of the radiation emitted by a transient dipole depends on the length of the dipole and the effective wavelength of the excitation current. The effective wavelength in turn depends on the duration of the current waveform. 
The literature on the radiation produced by Hertizian dipoles is numerous, and it is sufficed to refer to several textbooks dealing with the subject such as Jackson [1] and Panofsky and Philips [2]. There is also a significant amount of literature on the radiation fields of oscillating long dipoles. A dipole working in frequency domain is called "long" because the wavelength associated with the current oscillations is either comparable or shorter than the length of the dipole [3]. Even in the case of long transient dipoles, a certain amount of information on their radiation fields is available in the literature [4]. In the case of transient dipoles, the adjective "long" shows that the effective wavelength of the current exciting the dipole is either comparable to or shorter than the length of the dipole, or the duration of the current is either comparable to or shorter than the travel time of the current along the dipole. The current evolution in these dipoles has to be described by current pulses that travel from one end of the dipole to the other. Theories on long dipoles are developed mainly in connection with lightning research, where currents are assumed to propagate along long lightning channels [4]. Irrespective of these advances, however, the way in which the momentum transported by the radiation fields of a dipole varies with the duration of the excitation current and the length of the dipole has never been investigated in the literature. The goal of this paper is to fill this gap in our knowledge concerning dipole radiation.

In this paper we will consider the momentum transported by the radiation fields of a long transient dipole. We will consider the general case where the characteristic wavelength of the current pulse propagating along the dipole can take any value with respect to the length of the dipole.

\section{Radiation Fields Produced by a Transient Dipole}

\subsection{Transient Hertzian Dipole}

The features of radiation fields generated by Hertzian dipoles are described in any textbook on electromagnetic theory. Consider a dipole, of length $L$ and radius $a$, located along the $z$-axis. The relevant geometry is shown in Figure 1a. Let us denote the excitation current of the dipole by $i(t)$. In our study, we will consider a Gaussian current pulse (see Section 2.2 for more details). Since our interest here is to obtain general results concerning the connection between the energy and the momentum transported by the radiation fields of transient dipoles, the Gaussian pulse is more appropriate. Moreover, the frequency spectrum of a Gaussian current pulse is also a Gaussian. However, as we will show later, the results obtained are also valid for other current signatures. In a Gaussian current pulse, the amplitude decays by $e^{-2}$ when one moves $2 \sigma$ away in either direction in time from the peak value. Thus, the duration $\tau$ of the Gaussian pulse is about $4 \sigma$. The characteristic wavelength (the wavelength associated with the frequency when the amplitude of the frequency spectrum decays to $e^{-2}$ of its peak value), $\lambda_{e}$, associated with the current is approximately equal to $\tau c$, where $c$ is the speed of light in free space [5]. In the case of a Hertzian dipole, the effective wavelength should satisfy the conditions $\lambda_{e}>>a$ and $\lambda_{e}>>L$. When the distance to the point of observation $r$ is much larger than the effective wavelength $\lambda_{e}$ of the dipole, its electromagnetic fields become pure radiation. The radiation fields generated by a Hertzian dipole are given by $[1,2]$

$$
\begin{gathered}
\mathbf{E}_{\theta}=\frac{L}{4 \pi \varepsilon_{0} c^{2} r} \sin \theta \frac{d i(t-r / c)}{d t} \mathbf{a}_{\theta} \\
\mathbf{H}_{\varphi}=\frac{L}{4 \pi c r} \sin \theta \frac{d i(t-r / c)}{d t} \mathbf{a}_{\varphi} \\
\mathbf{D}_{\theta}=\frac{L}{4 \pi c^{2} r} \sin \theta \frac{d i(t-r / c)}{d t} \mathbf{a}_{\theta}
\end{gathered}
$$

One can use this set of equations to evaluate the energy and momentum transported by Hertzian dipoles. 


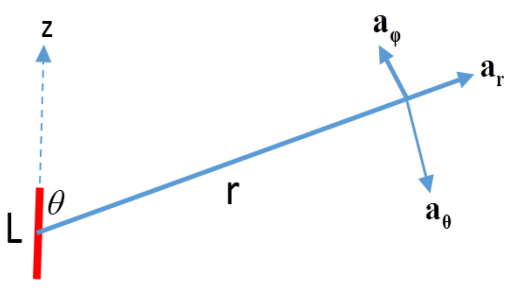

(a)

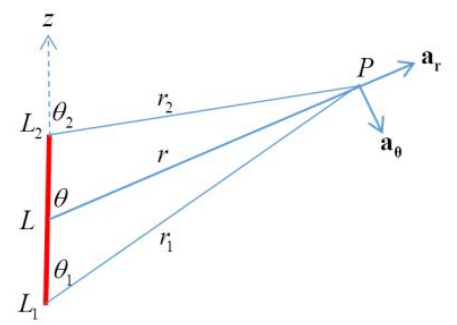

(b)

Figure 1. Geometry relevant to the derivation of equations presented in this paper. (a) Hertzian dipole; (b) long dipole. Observe that in defining the unit vectors $\mathbf{a}_{r}, \mathbf{a}_{\boldsymbol{\theta}}$, and $\mathbf{a}_{\varphi}$ we are using a spherical coordinate system with the centre of the dipole located at the origin of coordinate system. In this coordinate system $r$ is the radial distance, $\theta$ is the polar angle, and $\varphi$ is the azimuthal angle. $\mathbf{a}_{r}, \mathbf{a}_{\theta}$, and $\mathbf{a}_{\varphi}$ are unit vectors in the direction of increasing radial distance, polar angle and azimuthal angle, respectively.

\subsection{Transient Long Dipole}

The geometry relevant to the problem under consideration is shown in Figure 1b. A current pulse is initiated at end $L_{1}$ of the dipole and travels with constant speed towards the end $L_{2}$ of the dipole. The constant speed of propagation of the current pulse is assumed to be equal to the speed of light in free space. This indeed is the speed of propagation of current pulses in antennas located in free space $[1,3]$. The length of the dipole is denoted by $L$ and it is located along the $z$-axis. Thus, the direction of propagation of the current is aligned along the $z$-axis. The point of observation is denoted by $P$ and the distances to the point of observation and its angular position with respect to the ends of the dipole are shown in Figure $1 \mathrm{~b}$. Since we are interested in the net momentum transported by the emitted electromagnetic radiation, it is sufficed to evaluate the radiation fields generated by the dipole. The signature of the radiation field generated by a dipole which is excited by a transient current consists of two pulses, one generated during the initiation of the current pulse at $L_{1}$ and the other during the termination of the current pulse at $L_{2}[6]$.

In the present study, the temporal variation of the current pulse propagation along the dipole is represented by a Gaussian curve, which can be described mathematically by the following analytical function:

$$
i(t)=i_{0} \exp \left(-\frac{(t-4 \sigma)^{2}}{2 \sigma^{2}}\right)
$$

Note that, for the ease of calculation, the Gaussian current pulse is shifted forward in time by $4 \sigma$, where $\sigma$ is the standard deviation. This pulse, with the amplitude normalized to unity, is shown in Figure 2. In the calculation, it is assumed that $\sigma=10 \mathrm{~ns}$. Observe, in this diagram, that the duration of the current pulse $\tau$ is about $4 \sigma$ (see Section 2.1).

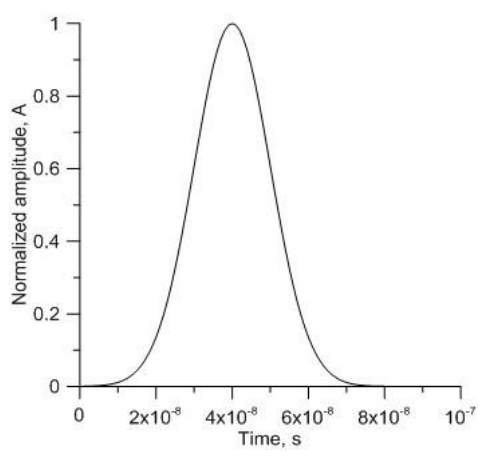

Figure 2. The normalized Gaussian current pulse with standard deviation $\sigma=10$ ns. 
An expression for the radiation field produced by the propagation current pulse at a distant point $\left(r_{1} \approx r_{2} \approx r ; \theta_{1} \approx \theta_{2} \approx \theta ; r \gg L\right)$ where the field is pure radiation is given by [6]. It can be described mathematically by the following set of equations:

$$
\begin{gathered}
\mathbf{E}_{\mathrm{rad}}(t, \theta)=\frac{i(t-r / c) \sin \theta}{4 \pi \varepsilon_{0} c r}\left[\frac{1}{1-\cos \theta}\right] \mathbf{a}_{\theta} \text { for } r / c<t<r / c+\delta_{1} \\
\mathbf{E}_{\mathrm{rad}}(t, \theta)=\frac{\sin \theta}{4 \pi \varepsilon_{0} c r}\left[\frac{i(t-r / c)}{1-\cos \theta}-\frac{i\left(t-r / c-\delta_{1}\right)}{1-\cos \theta}\right] \mathbf{a}_{\theta} \text { for } t>r / c+\delta_{1}
\end{gathered}
$$

where

$$
\delta_{1}=\frac{L}{c}-\frac{L \cos \theta}{c}
$$

Note that $\delta_{1}$ is the delay in the emission of radiation from the two ends. The field components $\mathbf{H}_{\varphi}$ and $\mathbf{D}_{\theta}$ can be obtained from the equations given above using the relationships $\left|\mathbf{H}_{\varphi}\right|=\varepsilon_{0} c\left|\mathbf{E}_{\theta}\right|$ and $\mathbf{D}_{\boldsymbol{\theta}}=\varepsilon_{0} \mathbf{E}_{\boldsymbol{\theta}}$. It is possible to show that when the conditions $\lambda_{e}>l$ and $\lambda_{e}>>a$ are satisfied, Equations $(5 a, b)$ reduce to the Hertzian dipole electric field given by Equation (1) [7].

The radiation field generated by a long dipole (with $\tau /(L / c)=0.01$ ) along the direction $\theta=20^{\circ}$ is shown in Figure 3. Observe that the radiation field amplitude is normalized to unity. The two radiation bursts that one can see in the figure are generated at the initiation and termination of the current pulse.

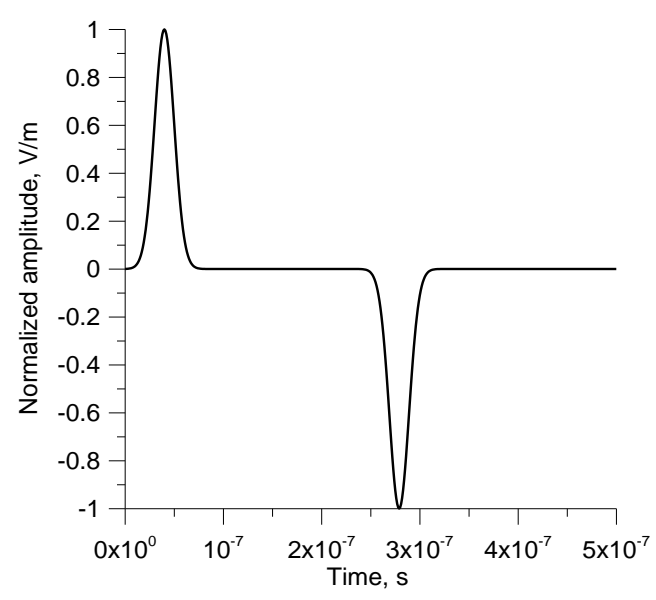

Figure 3. Normalized radiation field generated along the direction $\theta=20^{\circ}$ at a distant point by a long dipole. The dipole is excited by a Gaussian current pulse. In the calculation, $\sigma=10 \mathrm{~ns}$ and $\tau /(L / c)=0.01$.

\section{The Energy and Momentum of the Radiation Field}

The energy density generated by an electromagnetic field is given by the Poynting vector $\mathbf{S}$ which in turn is defined by $[1,2]$

$$
\mathbf{S}=\mathbf{E} \times \mathbf{H}
$$

This gives the power transmitted by the electromagnetic wave across a unit area located perpendicular to the direction of propagation of the wave. The momentum density associated with this electromagnetic field $\mathbf{P}$ is given by $[1,2]$

$$
\mathbf{P}=\frac{1}{c} \mathbf{E} \times \mathbf{H}
$$


This expression gives the linear momentum transferred per unit time by the wave across a unit area located perpendicular to the direction of propagation of the wave. In the above equations, $\mathbf{E}$ and $\mathbf{H}$ are the electric flux density and magnetic field intensity, respectively.

\subsection{Transient Hertzian Dipole}

The Poynting vector and the momentum density vector associated with the Hertzian dipole is given by

$$
\begin{aligned}
& \mathbf{S}(t, \theta)=\frac{L^{2} \sin ^{2} \theta}{16 \pi^{2} \varepsilon_{0} c^{3} r^{2}}\left(\frac{d i(t-r / c)}{d t}\right)^{2} \mathbf{a}_{r} \\
& \mathbf{P}(t, \theta)=\frac{L^{2} \sin ^{2} \theta}{16 \pi^{2} \varepsilon_{0} c^{4} r^{2}}\left(\frac{d i(t-r / c)}{d t}\right)^{2} \mathbf{a}_{r}
\end{aligned}
$$

The total energy radiated by the dipole is given by

$$
U=\frac{L^{2}}{16 \pi^{2} \varepsilon_{o} c^{3}} F \int_{0}^{2 \pi} \int_{0}^{\pi} \sin ^{3} \theta d \theta d \varphi
$$

where

$$
F=\int_{0}^{\infty}\left(\frac{d i(t)}{d t}\right)^{2} d t
$$

One can easily show that, due to symmetry, net momentum transported by the radiation field in any given direction is zero. For example, the net momentum transported in the $z$-direction by a Hertzian dipole is given by

$$
P_{z}=\frac{L^{2}}{16 \pi^{2} \varepsilon_{0} c^{4}} F \int_{0}^{2 \pi} \int_{0}^{\pi} \sin ^{3} \theta \cos \theta d \theta d \varphi
$$

Due to symmetry, the value of the above integral is zero. In other words, the ratio $P_{z} /(\mathrm{U} / \mathrm{c})$ for a Hertzian dipole is zero. In the sections to follow, we will show that this ratio departs from zero as the dipole changes from a Hertzian to a long dipole.

\subsection{Long Transient Dipole}

Using the expressions for the electric radiation fields given in Equation (5), and noting that $\mathbf{H}=\mathbf{E} / \mu_{0} c$ for radiation fields, we obtain

$$
\begin{gathered}
\mathbf{S}(t, \theta)=\frac{i^{2}(t-r / c) \sin ^{2} \theta}{16 \pi^{2} \varepsilon_{0} c r^{2}} \frac{1}{[1-\cos \theta]^{2}} \mathbf{a}_{r} \text { for } r / c<t<r / c+\delta_{1} \\
\mathbf{S}(t, \theta)=\frac{\sin ^{2} \theta}{16 \pi^{2} \varepsilon_{0} c r^{2}}\left[\frac{i(t-r / c)}{1-\cos \theta}-\frac{i\left(t-r / c-\delta_{1}\right)}{1-\cos \theta}\right]^{2} \mathbf{a}_{r} \text { for } t>r / c+\delta_{1}
\end{gathered}
$$

and

$$
\begin{gathered}
\mathbf{P}(t, \theta)=\frac{i(t-r / c)^{2} \sin ^{2} \theta}{16 \pi^{2} \varepsilon_{0} c^{2} r^{2}} \frac{1}{[1-\cos \theta]^{2}} \mathbf{a}_{r} \text { for } r / c<t<r / c+\delta_{1} \\
\mathbf{P}(t, \theta)=\frac{\sin ^{2} \theta}{16 \pi^{2} \varepsilon_{0} c^{2} r^{2}}\left[\frac{i(t-r / c)}{1-\cos \theta}-\frac{i\left(t-r / c-\delta_{1}\right)}{1-\cos \theta}\right]^{2} \mathbf{a}_{r} \text { for } t>r / c+\delta_{1}
\end{gathered}
$$

The energy radiated across a unit area in any given direction $\theta$ is given by

$$
u(\theta)=\int_{0}^{\infty} S(t, \theta) d t
$$


The total energy radiated by the system can be obtained by integrating the Poynting vector over a spherical region. That is, the total energy dissipated by the system is given by

$$
U=\int_{0}^{2 \pi} \int_{0}^{\pi} u(\theta) \sin \theta r^{2} d \theta d \varphi
$$

The net momentum transported by the electromagnetic field through a unit area in the direction $\theta$ is given by

$$
p(\theta)=\int_{0}^{\infty} P(t, \theta) d t
$$

Due to the angular symmetry of the emitted radiation, the $x$ and $y$ components of this momentum will add up to zero, leaving behind only the $z$ component. The net momentum transported in the $z$ - direction by the electromagnetic field is given by

$$
P_{z}=\int_{0}^{2 \pi} \int_{0}^{\pi} p(\theta) \sin \theta \cos \theta r^{2} d \theta d \varphi
$$

Since this is the only component of the net momentum transported by the radiation, we will drop the subscript $\mathrm{z}$ and write it simply as $P$.

Consider a transient electromagnetic field emitted in a direction $\theta$ by a dipole. If the electromagnetic field is confined only to direction $\theta$, then the total energy radiated $U$ and the total momentum $P$ transported by the electromagnetic field are related through Equations (6) and (7) as

$$
P=U / c
$$

In the case of a normal dipole, the net momentum transported by the radiation field departs from this equation because the radiation is not directed in one particular direction. However, as we will show in this paper, as the effective wavelength $\lambda_{e}$ of the current becomes comparable to or shorter than the length of the dipole (or the duration of the current pulse becomes comparable to or shorter than the travel time of the current pulse across the dipole), the radiation becomes more and more directional and the relationship between the momentum transfer and the total energy approaches $P=U / c$ (note that $P$ is the $z$-component of the transferred momentum). This will be investigated in the next section.

\section{The Total Electromagnetic Energy Radiated and the Total Electromagnetic Momentum Transported by a Long Dipole-General Case}

In order to take into account the radiation fields generated by long dipoles excited by current waveforms of different durations, let us define a parameter $\beta$ as

$$
\tau=\beta L / c
$$

In Equation (20), $\tau$ is the duration of the pulse and $L / c$ is the time taken by the current pulse to propagate the length of the dipole. In the case of the Gaussian pulse, $\tau=4 \sigma$ and, therefore, $\beta$ and $\tau$, are connected through the equation $\sigma=\beta L / 4 c$. As $\beta$ decreases, the duration of the current pulse becomes shorter and shorter in comparison to the time given by $L / c$.

The way in which the radiated energy of a dipole varies as a function of $\tau$ and $\beta$ has been studied previously by Cooray and Cooray [8]. They showed that the total energy radiated by a long dipole excited by a Gaussian current pulse can be written as

$$
U / c=q^{2} F_{u}(\beta) / \tau
$$


In the above equation, $F_{u}(\beta)$ is a function that depends only on $\beta$, and $q$ is the charge associated with the Gaussian current pulse. That is:

$$
q=\int_{0}^{\infty} i(t) d t
$$

Since the time dependence of the current, which appeared in the expressions for both the energy and the momentum transfer, is the same except for a geometrical factor that arises due to the presence of $\cos \theta$ in the integration that appears in Equation (12), the net momentum transported by the radiation field can be written as

$$
P=q^{2} F_{p}(\beta) / \tau
$$

Thus, the ratio of the total momentum and the total energy (divided by the speed of light) transported by the dipole is given by

$$
\frac{P}{U / c}=\frac{F_{p}(\beta)}{F_{u}(\beta)}
$$

This ratio depends only on the parameter $\beta$. In the case where $\beta \ll 1$ one can derive an analytical expression for the above ratio. This is done in the next section.

\section{The Total Electromagnetic Energy Dissipated by the Long Dipole and the Total Momentum Transported by the Radiation-Case When $\beta \ll 1$}

Observe that, as given by Equation ( $5 \mathrm{c})$, the delay between the two radiation pulses generated by the dipole during current initiation and termination is equal to $(1-\cos \theta) L / c$. For very small angles, the two pulses overlap. As the angle $\theta$ increases from zero, the separation between the radiation field pulses increases; when it reaches a critical value, say $\theta_{0}$, the two pulses separate from each other completely. This critical angle is given by

$$
\left(1-\cos \theta_{0}\right) \frac{L}{c}=\tau
$$

Substituting for $\tau$ in terms of $\beta$, we obtain

$$
\cos \theta_{0}=(1-\beta)
$$

As the value of $\beta$ decreases, the angle $\theta_{0}$ decreases; for very small values of $\beta$, it becomes almost equal to zero. In such cases, almost all the energy generated by the dipole is confined to the spatial region where $\theta>\theta_{0}$. As pointed out earlier, in this region of space the radiation field consists of two separate pulses, each with a signature identical to that of the current pulse. Thus, the total energy radiated by the dipole can be approximated by

$$
U=\frac{2\left[\int_{0}^{\infty} i^{2}(t-r / c) d t\right]}{16 \pi^{2} \varepsilon_{0} c} \int_{0}^{2 \pi} \int_{\theta_{0}}^{\pi} \frac{\sin ^{3} \theta}{[1-\cos \theta]^{2}} d \theta d \varphi
$$

The factor 2 in the above equation comes from the fact that there are two identical radiation pulses, each with a time signature identical to that of the current waveform. The expression for the total momentum transported by the radiation field is given similarly by the expression

$$
P=\frac{2\left[\int_{0}^{\infty} i^{2}(t-r / c) d t\right]}{16 \pi^{2} \varepsilon_{0} c^{2}} \int_{0}^{2 \pi} \int_{\theta_{0}}^{\pi} \frac{\sin ^{3} \theta \cos \theta}{[1-\cos \theta]^{2}} d \theta d \varphi
$$


The spatial integrals in Equations (27) and (28) can be evaluated without much difficulty. Furthermore, when one evaluates the ratio $P /(U / c)$, the time integral of the square of the current cancels off. Thus, the final result is given by

$$
\frac{P}{U / c}=\frac{\log (1 / \beta)-1.3}{\log (1 / \beta)-0.305} \quad \beta \ll 1
$$

Note in the above equation that the ratio becomes 1 as $1 / \beta$ goes to infinity.

\section{The Relationship between the Total Energy Dissipated and the Total Momentum Transported by the Long Dipole Field}

Let us consider the ratio $P /(U / c)$ for a dipole. This ratio is given by Equation (24) and, for the case $\beta \ll 1$, by Equation (29). Again, we remind the reader that $P$ stands for the net momentum transferred by the radiation field in the $z$-direction. A plot of $P /(U / c)$ as a function of $1 / \beta$ is shown in Figure 4. The ratio calculated using the approximate expression given by Equation (29) is shown in the same diagram by a dotted line. Observe that this ratio depends on the value of $1 / \beta$. Let us study how this ratio varies with this parameter. For small values of $1 / \beta$, the ratio $P /(U / c)$ is significantly less than unity, and this indicates that the radiation is not strongly directional. Indeed, as seen earlier, for a Hertzian dipole $1 / \beta \ll 1$ and the ratio $P /(U / c)$ is equal to zero. However, as $1 / \beta$ increases, the ratio increases and its value asymptotically approaches unity. For values of $1 / \beta$ larger than about $10^{5}-10^{6}$, this ratio is almost equal to unity. This shows that as $1 / \beta$ increases, the radiation becomes more and more directional and it aligns along the $z$-axis (or along the axis of the dipole). When $1 / \beta \geq 10^{5}$, the total momentum transported by the field is almost equal to $U / c$, where $U$ is the total energy radiated. In other words, for $1 / \beta \gg 1$ the radiation emitted by the dipole can be treated as almost unidirectional.

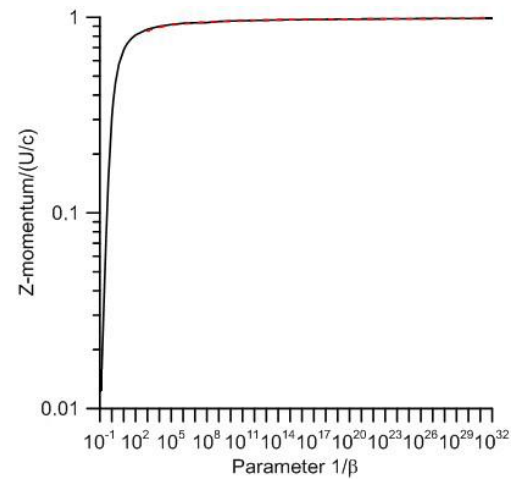

Figure 4. The variation of the ratio of $z$-momentum to $U / c$ as a function of $1 / \beta$. The solid line is calculated using Equation (24) and the dashed line by using Equation (29).

The results presented here are obtained for a Gaussian current pulse. However, observe that the ratio $P /(U / c)$ for large $1 / \beta$ is independent of the current. Thus, the results obtained above-which show that the radiation becomes more and more unidirectional as $1 / \beta$ increases-is valid also for other current waveforms. Actually, a similar effect can also be observed in dipole antennas where sinusoidal currents are oscillating. When the length of the antenna to the wavelength ratio (i.e., $L / \lambda$ is very small), the radiation as a function of $\theta$ has a broad peak, as in the case of sinusoidal Hertzian dipole [3]. However, as the ratio $L / \lambda$ increases, the radiation becomes more and more directional; for an antenna located along the $z$-axis, the radiation direction aligns along the $z$-axis $[3,8]$. This is exactly what happens when $1 / \beta$ increases in the long dipole. Note that the equivalent wavelength, $\lambda_{e}$, for a current pulse of duration $\tau$ is about $c \tau$. Thus, the ratio $L / \lambda_{e}$ is equal to $L / \tau c$, and this in turn is equal to $1 / \beta$. This shows that the reason for the increase in directivity of the radiation as $1 / \beta$ increases can also be understood by appealing to radiation generated by dipole antennas working in frequency domain. 


\section{Discussion}

The results presented in this paper can be utilized in a hypothetical experiment to derive an interesting property associated with dipole radiation. Consider an electromagnetic dipole completely at rest. Its location is unknown. At a given time, the dipole emits a burst of electromagnetic radiation whose duration is $\Delta t$. The task is to measure the momentum and the location of the dipole by using the emitted electromagnetic radiation. Let us assume that the emission mechanism of radiation satisfies the condition that the total momentum transported by the dipole electromagnetic field is given by $U / c$ where $U$ is the total energy associated with the electromagnetic radiation (i.e., $1 / \beta \geq 10^{5}$ ). The law of momentum conservation dictates that a momentum of equal magnitude but of opposite direction is transferred to the dipole by the outgoing radiation field. Let $\Delta U$ represents the uncertainty in the emitted energy. Then, the uncertainty in the momentum of the dipole $\Delta P$ is equal to $\Delta U / c$. This is the case because $P=U / c$. If the duration of the radiation emitted by the dipole is $\Delta t$, the effective wavelength associated with the radiation is $c \Delta t$. Now, when an object is illuminated by electromagnetic radiation, the location of the object cannot be specified to an accuracy better than the wavelength of the radiation [9]. Thus, the uncertainty in the position of the dipole in the $z$-direction, $\Delta z$, is equal to $c \Delta t$. Since the uncertainty in the position and the momentum of any object should satisfy the Heisenberg's uncertainty principle we can write

$$
\Delta P \Delta z \geq \frac{h}{4 \pi}
$$

In the above equation, $h$ is the Plank constant. Substituting $\Delta P=\Delta U / c$ and $\Delta z=c \Delta t$ in Equation (30) we obtain

$$
\Delta U \Delta t \geq \frac{h}{4 \pi}
$$

In our case, the condition $P=U / c$ is approximately satisfied by the radiation fields when the value of $1 / \beta$ is larger than about $10^{5}$. Thus, for dipoles working in time domain where the condition $1 / \beta \geq 10^{5}$ is satisfied, the emitted radiation should satisfy approximately the condition given by Equation (31). This is an interesting result, which shows that dipole fields, when excited by fast current pulses, satisfy a time-energy uncertainty relationship as given by Equation (31). Indeed, Cooray and Cooray [8] used this relationship to show that the smallest charge that can radiate in an antenna working in time domain is equal to the electronic charge.

In order to be treated as a Hertzian dipole, the effective wavelength associated with the radiation $\lambda_{e}$ of the dipole should satisfy the conditions $\lambda_{e}>>a$ and $\lambda_{e}>>L$, where $a$ and $L$ are the radius and length of the dipole, respectively. In the case of a long dipole, we relax the second condition, and this makes it necessary to consider the propagation effects of the current along the dipole. However, even in the case of a long dipole, we still have to satisfy the condition $\lambda_{e}>>a$. Thus, the results obtained for a long dipole are valid, provided this condition is satisfied. This shows that the possible values of $1 / \beta$ are bound on one side by the smallest radius of the conductor that can be realized in nature and on the other side by the longest dipole that can be realized in nature (i.e., $L \ll L_{u}$ where $L_{u}$ is the radius of the universe).

In this paper, we have considered an idealized situation in which current pulses are assumed to propagate along the long dipole with speed of light and without attenuation. This idealization was warranted here because the goal of the study reported in this paper was to understand the connection between the energy and momentum transported by the long dipole radiation. However, the application of this procedure in more practical situations needs further consideration. For example, dipole antennas are constructed in practice by locating vertical conductors over perfectly conducting ground. In this case, the long dipole acts as a vertical transmission line. Pulses propagating along vertical conductors located over a ground plane are affected by the finite conductivity of the material of the conductor and by the effects of radiation damping [1]. The calculations conducted by Baba and Rakov [10] show that the current pulses propagating along such conductors can attenuate as they propagate along them. Moreover, close to the ground end of the conductors, even the speed of 
propagation of the current pulses is slightly less than the speed of light. At present, we are investigating these effects to extract the conditions under which the ideal assumptions made in the present paper, namely propagation of current pulses without attenuation, are valid. The results obtained by us at present using the mathematical equations developed by $\mathrm{Wu}$ [11] and Chen [12] for pulse propagation along vertical conductors located over a ground plane, indicate that the ideal conditions assumed in the present paper are valid for values of $1 / \beta$ less than about $10^{6}-10^{7}$. Further research work is necessary to confirm this result.

Another natural phenomenon that acts as a dipole located over a ground plane is the lightning return stroke. Experimental data show that the return stroke can be treated as a current pulse propagating along a more or less vertical channel with an average speed of propagation of about $(1-2) \times 10^{8} \mathrm{~m} / \mathrm{s}$ while undergoing attenuation. The actual front speed decreases with increasing height [13]. In reality, the ground is finitely conducting and the radiation fields are affected by the propagation losses. Research work is underway to investigate the momentum transferred by radiation fields of lightning return strokes and how it is related to the total energy radiated by them. Furthermore, how the magnitude of the radiated momentum and its relationship to total radiated energy vary as a function of return stroke parameters-such as current amplitude, current signature, speed of propagation, and current attenuation along the channel-are under investigation. We hope to present the results of these investigations in the near future.

\section{Conclusions}

The paper describes the net momentum transported by the radiation emitted by a transient dipole working in time domain. In the dipole a current is initiated at one end and propagates towards the other end where it is absorbed. The net momentum associated with the radiation is directed along the axis of the dipole. It is shown that as the duration of the excitation current of the dipole decreases with respect to the travel time of the current along the dipole, $P \rightarrow U / c$ where $P$ is the net momentum associated with the radiation, $U$ is the total energy radiated by the dipole and $c$ is the speed of light in free space. When the ratio of the duration of the current to the travel time of the current along the dipole decreases below about $10^{-5}$, the net momentum and the radiated energy are connected by the relationship $P \approx U / c$. Due to momentum conservation, the radiating dipole experiences a momentum of equal magnitude but in the opposite direction. The results show that under the conditions when $P \approx U / c$ is satisfied, the radiation fields satisfy the relationship $\Delta t \Delta U \geq h / 4 \pi$ where $\Delta t$ is the duration of the radiation, $\Delta U$ is the uncertainty in the radiated energy, and $h$ is the Plank constant.

Acknowledgments: The research presented in this paper is supported by the fund from B. John F. and Svea Andersson donation at Uppsala University.

Author Contributions: The research problem was suggested by the first author. Both authors contributed equally in carrying out this research work and in writing the paper. Both the authors have read and approved the final manuscript.

Conflicts of Interest: The authors declare no conflict of interest.

\section{References}

1. Jackson, J.D. Classical Electrodynamics; Wiley: New York, NY, USA, 1962.

2. Panofsky, W.K.H.; Phillips, M. Classical Electricity and Magnetism; Addison-Wesley: Reading, MA, USA, 1962.

3. Balanis, C.A. Antenna Theory: Analysis and Design; Harper \& Row Publishers: New York, NY, USA, 1982.

4. Thottappillil, R. Calculation of electromagnetic fields from lightning. In Lightning Electromagnetics; Cooray, V., Ed.; IET: Stevenage, UK, 2014.

5. Bracewell, R. The Fourier Transform and Its Applications, 3rd ed.; McGraw-Hill: New York, NY, USA, 1999; pp. 98-101.

6. Cooray, V.; Cooray, G. The Electromagnetic fields of an accelerating charge: Applications in lightning return-stroke models. IEEE Proc. 2010, 52, 944-955. [CrossRef] 
7. Cooray, G.; Cooray, V. Electromagnetic fields of a short electric dipole in free space-Revisited. Prog. Electromagn. Res. 2012, 131, 357-373. [CrossRef]

8. Cooray, V.; Cooray, G. On the Remarkable Features of the Lower Limits of Charge and the Radiated Energy of Antennas as Predicted by Classical Electrodynamics. Atmosphere 2016, 7, 64. [CrossRef]

9. Bransden, B.H.; Joachain, C.J. Introduction to Quantum Mechanics; Longman Scientific \& Technical: London, UK, 1989.

10. Baba, Y.; Rakov, V. On the mechanism of attenuation of current waves propagating along a vertical perfectly conducting wire above ground: Application to lightning. IEEE Trans. Electromagn. Compat. 2005, 47, 521-532. [CrossRef]

11. Wu, T.T. Transient response of a dipole antenna. J. Math. Phys. 1961, 2, 892-894. [CrossRef]

12. Chen, K.C. Transient response of an infinite cylindrical antenna. IEEE Trans. Antennas 1983, 35, 562-573. [CrossRef]

13. Cooray, V. The Mechanism of the lightning flash. In The Lightning Flash, 2nd ed.; Cooray, V., Ed.; Institution of Engineering and Technology (IET): Stevenage, UK, 2008.

(C) 2016 by the authors; licensee MDPI, Basel, Switzerland. This article is an open access article distributed under the terms and conditions of the Creative Commons Attribution (CC-BY) license (http://creativecommons.org/licenses/by/4.0/). 